\title{
The evolution of the PCPT from clinical to molecular analyses
}

\author{
Ashraful Hoque
}

In the Prostate Cancer Prevention Trial (PCPT), which randomized 18,882 men with normal digital rectal examination (DRE) results and prostatespecific antigen (PSA) levels, finasteride significantly reduced prostate cancer risk by $24.8 \%$ but also increased high-grade disease (Gleason score 27) compared with placebo (Thompson IM et al. [2003] N Engl J Med 349: 215-224). The prevalence of prostate cancer, including high-grade tumors, was $15.2 \%$ among men with 'normal' ( $\leq 4 \mathrm{ng} / \mathrm{ml}$ ) PSA levels (Thompson IM et al. [2004] $N$ Engl J Med 350: 2239-2246); in addition, finasteride increased the sensitivity of PSA for detecting prostate cancer when compared with placebo (Thompson IM et al. [2006] J Natl Cancer Inst 98: 1128-1133). Whether the increased highgrade disease associated with finasteride was an artifact is under intensive pathological analysis and is one of several questions being addressed by a long-term follow-up study of men who developed prostate cancer during the PCPT.

Uniquely, the PCPT was designed to ascertain its primary endpoint (detection of prostate cancer) via a prostate biopsy regardless of PSA status and DRE result at the end of the study. The PCPT therefore provides a biorepository with a large number of biopsy-proven control (no cancer) samples that can be used for molecular analyses. Over 3,100 men received an interim, or 'for-cause', biopsy, prompted primarily by PSA levels $>4.0 \mathrm{ng} / \mathrm{ml}$ and/or an abnormal DRE. Almost 12,000 men were eligible for the end-of-study biopsy, and $62 \%$ complied. The PCPT established a large bank of biopsy tissue, prostatectomy specimens, and serum, plasma and blood cell samples, which were collected after endpoint events, at interim time points, and at study end. These specimens and clinical data offer a unique opportunity to study the risk of prostate cancer and the pharmacoecogenetics (environmental, metabolic and genetic factors) of finasteride for inhibiting prostate carcinogenesis.

Molecular studies of the PCPT are currently underway and use a nested case-control design

\section{Molecular \\ studies of \\ the PCPT... \\ should allow \\ personalized \\ prevention \\ of prostate \\ cancer with \\ finasteride}

A Hoque is Assistant

Professor in the

departments of

Clinical Cancer

Prevention and

Epidemiology, MD

Anderson Cancer

Center, TX, USA.

Competing interests

The author has declared

associations with the

Prostate Cancer Prevention

Trial and the National

Cancer Institute. See the

article online for full details

of the relationship.

www.nature.com/clinicalpractice doi:10.1038/ncponc0653 to assess important factors and biological mechanisms underlying prostate cancer risk and the effects of finasteride treatment. Molecular factors under investigation include single nucleotide polymorphisms of genes involved in steroid metabolism, oxidative stress and DNA repair; levels of serum androgen and estrogens; dietary factors; insulin-like growth factors; somatic mutations of steroid metabolic genes; and inflammation. These analyses should allow a more precise definition of prostate cancer risk, which is currently defined only by age, ethnicity and family history risk factors. Studies comparing the molecular characteristics of patients with high-grade and low-grade disease in both the finasteride and placebo arms will address whether finasteride truly increased high-grade prostate cancer incidence.

Previous molecular and other epidemiologic studies have used negative controls based on a 'normal' PSA and/or DRE, which were associated with a substantial $(>15 \%)$ prevalence of prostate cancer in the PCPT. Major limitations of the previous studies include detection bias and misclassified controls; however, owing to prospectively collected negative (no cancer) controls, PCPT molecular studies promise to provide the most accurate assessments of prostate cancer risk ever made.

Even though finasteride reduced prostate cancer risk by a significant amount ( $25 \%)$ in the PCPT, it has not changed standards of preventive care because we have no current means for determining which men are at the highest risk, which are most likely to benefit from finasteride, and which are least likely to develop high-grade disease if they use finasteride. Molecular studies of the PCPT are addressing these issues and should allow personalized prevention of prostate cancer with finasteride. Besides providing great insight into prostate cancer risk and the role of finasteride in prostate cancer prevention, molecular studies of the PCPT will also provide a template for risk and pharmacoecogenetic modeling in other major organ sites. 